\title{
Tumor Mesenchymal Stem-Like Cell as a Prognostic Marker in Primary Glioblastoma
}

\author{
Seon-Jin Yoon, ${ }^{1}$ Jin-Kyoung Shim, ${ }^{1}$ Jong Hee Chang, ${ }^{1}$ Ju Hyung Moon, ${ }^{1}$ \\ Tae-Hoon Roh, ${ }^{1}$ Kyoung Su Sung, ${ }^{1}$ Ji-Hyun Lee, ${ }^{1}$ Eui-Hyun Kim, ${ }^{1}$ Sun Ho Kim, \\ Yong-Kil Hong, ${ }^{2}$ Su-Jae Lee, ${ }^{3}$ Yong-Min Huh, ${ }^{4}$ and Seok-Gu Kang ${ }^{1}$ \\ ${ }^{1}$ Department of Neurosurgery, Brain Tumor Center, Severance Hospital, Yonsei University College of Medicine, \\ 50-1 Yonsei-ro, Seodaemun-gu, Seoul 120-752, Republic of Korea \\ ${ }^{2}$ Department of Neurosurgery, Seoul St. Mary's Hospital, The Catholic University of Korea College of Medicine, \\ 222 Banpo-daero, Seocho-gu, Seoul 137-701, Republic of Korea \\ ${ }^{3}$ Department of Life Science, Hanyang University, 17 Haendang-dong, Seongdong-gu, Seoul 133-791, Republic of Korea \\ ${ }^{4}$ Department of Radiology, Severance Hospital, Yonsei University College of Medicine, 50-1 Yonsei-ro, Seodaemun-gu, \\ Seoul 120-752, Republic of Korea \\ Correspondence should be addressed to Seok-Gu Kang; seokgu9@kornet.net
}

Received 4 November 2015; Revised 5 January 2016; Accepted 13 January 2016

Academic Editor: Abdel Kareem Azab

Copyright ( 2016 Seon-Jin Yoon et al. This is an open access article distributed under the Creative Commons Attribution License, which permits unrestricted use, distribution, and reproduction in any medium, provided the original work is properly cited.

\begin{abstract}
The isolation from brain tumors of tumor mesenchymal stem-like cells (tMSLCs) suggests that these cells play a role in creating a microenvironment for tumor initiation and progression. The clinical characteristics of patients with primary glioblastoma (pGBM) positive for tMSLCs have not been determined. This study analyzed samples from 82 patients with pGBM who had undergone tumor removal, pathological diagnosis, and isolation of tMSLC from April 2009 to October 2014. Survival, extent of resection, molecular markers, and tMSLC culture results were statistically evaluated. Median overall survival was 18.6 months, 15.0 months in tMSLCpositive patients and 29.5 months in tMSLC-negative patients $(P=0.014)$. Multivariate cox regression model showed isolation of tMSLC $(\mathrm{OR}=2.5,95 \% \mathrm{CI}=1.1 \sim 5.6, P=0.021)$ showed poor outcome while larger extent of resection $(\mathrm{OR}=0.5,95 \% \mathrm{CI}=0.2 \sim 0.8$, $P=0.011)$ has association with better outcome. The presence of tMSLCs isolated from the specimen of pGBM is associated with the survival of patient.
\end{abstract}

\section{Introduction}

Glioblastomas (GBMs) are generated by interactions between cancer stem cells (CSCs) and stroma [1-3]. The accumulation of molecular errors in CSCs initiates tumorigenesis, and these CSCs aggregate with stromal cells, which synergistically aggravate the disease $[4,5]$. Mesenchymal stem-like cells (MSLCs) have been isolated from normal brain $[6,7]$ and Lang et al. [8] presented the isolation of mesenchymal stem cells (MSCs) from glioma for the first time. In addition, tumor MSLCs (tMSLCs) have been isolated from several human brain tumors [2, 9-11], suggesting that these cells play a role in creating a microenvironment conducive to brain tumor initiation and progression $[2,12-14]$.
GBMs can be grouped into several subtypes, based on molecular markers, gene expression profiles [15-19], and chromosomal aberration [20, 21]. Based on their genetic characteristics, GBMs can be divided into four types, with the mesenchymal type having the poorest prognosis $[17,18,22]$. Several molecular markers have been shown to be related to survival benefits in patients with GBM, including O-6methylguanine-DNA methyltransferase (MGMT) methylation and the isocitrate dehydrogenase (IDH) 1/2 mutation [23-25]. The prognostic value of heterozygosity ( $\mathrm{LOH})$ at chromosomes $1 p$ and $19 q$, however, is unclear [26, 27]. Isolation of CSCs from primary GBM (pGBM) samples can also predict the natural course of pGBM [28]. Although tumor stromal cells were found to have a significant impact on 
patient survival $[29,30]$, the clinical significance of isolation of tMSLCs, a type of stromal cells, from pGBM stroma has not been determined.

We hypothesized that the presence of tMSLCs may aggravate the natural course of pGBM. This study therefore assessed whether the presence of tMSLCs in pGBM patients has an effect on patient survival and prognosis.

\section{Materials and Methods}

2.1. Patient Information. A total of 82 patients with pGBM who received standard therapy [31] at two institutions (Severance Hospital, Yonsei University College of Medicine, and Seoul St. Mary's Hospital, the Catholic University of Korea College of Medicine) from April 2009 to October 2014 were included in this study (Table 1). We followed up the cohort from previous report [11] and added new additional patients that were not included in that report [11]. Approval for harvest and investigation was obtained from the institutional review boards of the two institutions, and all patients provided written informed consent, as specified in the Declaration of Helsinki. Specimens for isolation of tMSLCs were collected in the operating theater from patients undergoing surgery. All surgical specimens were evaluated by two neuropathologists, who diagnosed each patient according to World Health Organization (WHO) classifications [32]. Survival, extent of resection, molecular markers, and tMSLC culture results were analyzed statistically. Inclusion criteria were as follows: the first pathologic diagnosis of primary glioblastoma patients with the standard Stupp protocol; radiation dose of 60 Gy fractionated by 30 times; and Stupp protocol within 2 weeks after the pathological diagnosis. Excluded patients met following criteria: recurring glioblastoma after previous surgery; gliosarcoma; nonstandard dose for temozolomide administration; hypofractionated radiotherapy; and poor hematologic profile that delayed normal course of standard treatment.

2.2. Initial Treatment. All patients underwent surgical resection, aimed at gross total resection of the tumor, followed by concurrent chemotherapy and radiotherapy and adjuvant chemotherapy [31]. Gross total tumor resection was defined as macroscopic removal of $100 \%$ or above of the tumor mass found on magnetic resonance (MR) T1 enhanced and T2 images $[33,34]$. Patients not suitable for total resection underwent subtotal resection, defined as removal of macroscopic tumor volume $\geq 90 \%$ but $<100 \%$, or partial resection, defined as removal of macroscopic tumor volume $<90 \%$. The extent of tumor resection was estimated and classified by the neurosurgeons and rechecked by postoperative review of MR imaging (MRI) scans. All patients received postoperative adjuvant radiotherapy with concomitant and adjuvant temozolomide (TMZ), as described previously [31]. Each patient was offered standard therapy [31] after pathologic confirmation. The only factors determining the continuation of standard treatment were patient tolerance (general condition, laboratory abnormalities such as hematologic problems), family agreement for the treatment, and patient survival. The correlation between molecular markers (MGMT methylation, p53, 1p LOH, 19q LOH, Ki67 index, and IDH1 mutation) and survival was analyzed statistically. MGMT methylation was assessed by polymerase chain reaction (PCR) and $\mathrm{LOH}$ at chromosomes $1 \mathrm{p}$ and $19 \mathrm{q}$ by fluorescent in situ hybridization (FISH).

2.3. Isolation of tMSLCs. tMSLCs with characteristics similar to MSCs have been isolated from brain tumor specimens [911]. Specimens from patients with GBM were obtained from the operating room. Briefly, cells were isolated during tumor removal using a mechanical dissociation method within $1 \mathrm{~h}$. Surgical specimens were minced and dissociated with a scalpel in minimal essential medium- $\alpha$ (MEM $\alpha$; Mediatech, Herndon, VA, USA) and passed through a series of cell strainers with a $100 \mu \mathrm{m}$ nylon mesh. Cell suspensions were washed twice in MEM $\alpha$ and then cultured in complete MSC medium consisting of MEM $\alpha, 10 \%$ fetal bovine serum (FBS; Gibco, Invitrogen), $2 \mathrm{mM}$ L-glutamine (Mediatech), and $1 \mathrm{x}$ antibiotic-antimycotic solution (Invitrogen, Carlsbad, CA, USA). After $24 \mathrm{~h}$, nonadherent cells were removed by washing twice with phosphate-buffered saline (PBS; Mediatech), and the adherent cells were cultured until they reached confluence. The cells were then trypsinized ( $0.25 \%$ trypsin with $0.1 \%$ EDTA) and subcultured at a density of $5 \times 10^{3}$ cells $/ \mathrm{cm}^{2}$. Isolated cells were evaluated for several mesenchymal features, including plastic adhesion, trilineage differentiation, the presence of typical MSC surface markers (positive for CD 105, CD 90, and CD 72 and negative for CD 45, CD 31, and NG2), and nontumorigenic behavior [11, 13, 35].

2.4. Statistical Analysis. The primary outcome measure was median overall survival (OS), defined as the interval from date of surgery confirming the diagnosis of pGBM to the date of last follow-up visit or death [36]. Immunohistochemical analysis of p53 expression was defined as immunopositivity when the areas with staining of $\geq 50 \%$ of cancer cells were found. Ki 67 index was defined as immunopositive when the stained area exceeded $10 \%$ or more. Among clinically deemed primary glioblastomas, 5 patients $(6 \%)$ had mutation on IDH1. They were not excluded from our study for comprehensive evaluation. Because of small number of patients with IDH1 mutation and previous reports about different clinical characteristics [37], they were not eligible for survival analysis and excluded from multivariate cox regression model. Survival was analyzed by the Kaplan-Meier method and compared by the log-rank test. Demographic characteristics were compared using Fisher's exact test or $t$-test. All statistical analyses were performed using SPSS 22 (IBM Korea, Seoul, Korea), with $P$ values less than 0.05 regarded as statistically significant.

\section{Results}

3.1. Patient Characteristics. Of the 82 patients with pGBM, 48 (59\%) were positive and 34 (41\%) negative for tMSLCs (Table 1), with no group differences in the extent of resection $(P=0.471)$, age $(P=0.683)$, and expression of specific molecular markers (IDH1, $P=0.642$; MGMT promoter, $P=0.653$; p53, $P=0.522$ for positivity $P=0.492$ for the percentage of immunohistochemistry; EGFR, $P=0.161$; $\mathrm{Ki}$ $67, P=0.739$ for the number of each of the immunostaining 
TABLE 1: Demographic and clinical characteristics of patients with pGBM.

\begin{tabular}{|c|c|c|c|}
\hline Characteristics & tMSLCs $(+)(N=48)$ & tMSLCs $(-)(N=34)$ & $P$ value \\
\hline Age (years) & & & 0.683 \\
\hline Median & 57.5 & 61.0 & \\
\hline Range & $28 \sim 85$ & $24 \sim 80$ & \\
\hline Age, no. (\%) & & & 0.899 \\
\hline$<50$ years, no. $(\%)$ & $9(19)$ & $6(18)$ & \\
\hline$\geq 50$ years, no. $(\%)$ & $39(81)$ & $28(82)$ & \\
\hline Gender & & & 0.110 \\
\hline Male, no. (\%) & $33(69)$ & $17(50)$ & \\
\hline Female, no. (\%) & $15(31)$ & $17(50)$ & \\
\hline Median survival (months) & 15.0 & 29.5 & 0.014 \\
\hline $95 \% \mathrm{CI}$ & $9.6 \sim 20.4$ & $11.9 \sim 47.1$ & \\
\hline Pathological diagnosis & pGBM & pGBM & \\
\hline Treatment & OP/Stupp & OP/Stupp & \\
\hline Extent of operation (patients) & & & 0.471 \\
\hline Gross total resection $(\geq 100 \%)$ & $29(60)$ & $19(56)$ & \\
\hline Subtotal resection $(90 \% \leq,<100 \%)$ & $18(38)$ & $12(35)$ & \\
\hline Partial resection $(<90 \%)$ & $1(2)$ & $3(9)$ & \\
\hline \multicolumn{4}{|l|}{ Molecular markers } \\
\hline IDH1 & & & 0.642 \\
\hline Wild type, no. (\%) & $39(91)$ & $26(96)$ & \\
\hline Mutation, no. (\%) & $4(9)$ & $1(4)$ & \\
\hline Missing data, no. (\%) & $5(10)$ & $7(21)$ & \\
\hline $1 \mathrm{p} 19 \mathrm{q}$ & & & 0.341 \\
\hline No codeletion, no. (\%) & $37(80)$ & $30(91)$ & \\
\hline Median survival (months) & 15.0 & 29.5 & 0.011 \\
\hline $95 \% \mathrm{CI}$ & $8.9 \sim 21.1$ & $9.1 \sim 50.0$ & \\
\hline Codeletion, no. (\%) & $9(20)$ & $3(9)$ & \\
\hline Median survival (months) & 12.9 & 9.3 & 0.886 \\
\hline $95 \% \mathrm{CI}$ & $0.8 \sim 25.0$ & $1.6 \sim 17.0$ & \\
\hline Missing data, no. (\%) & $2(4)$ & $1(3)$ & \\
\hline MGMT promoter & & & 0.653 \\
\hline Wild type, no. (\%) & $27(59)$ & $18(53)$ & \\
\hline Median survival (months) & 15.0 & $\mathrm{NA}$ & 0.122 \\
\hline $95 \% \mathrm{CI}$ & $8.8 \sim 21.2$ & NA & \\
\hline Methylated, no. (\%) & $19(41)$ & $16(47)$ & \\
\hline Median survival (months) & 18.6 & 34.1 & 0.164 \\
\hline $95 \% \mathrm{CI}$ & $6.2 \sim 31.0$ & $13.6 \sim 54.6$ & \\
\hline Missing data, no. (\%) & $2(4)$ & $0(0)$ & \\
\hline p53 & & & 0.522 \\
\hline IHC negative $(<50 \%)$, no. $(\%)$ & $23(77)$ & $13(65)$ & \\
\hline Median survival (months) & 13.7 & NA & 0.324 \\
\hline $95 \% \mathrm{CI}$ & $9.8 \sim 17.6$ & NA & \\
\hline IHC positive ( $\geq 50 \%)$, no. (\%) & $7(23)$ & $7(35)$ & \\
\hline Median survival (months) & 18.6 & NA & 0.704 \\
\hline $95 \% \mathrm{CI}$ & $9.9 \sim 27.3$ & NA & \\
\hline IHC mean \pm S.D. & $28.4 \pm 27.0$ & $33.9 \pm 28.8$ & 0.492 \\
\hline Range (\%) & $1.5 \sim 85$ & $2.5 \sim 90$ & \\
\hline Missing data, no. (\%) & $18(38)$ & $14(41)$ & \\
\hline \multicolumn{4}{|l|}{ EGFR } \\
\hline IHC mean \pm S.D. & $1.9 \pm 1.1$ & $2.3 \pm 0.9$ & 0.161 \\
\hline Range & $0 \sim 3$ & $0 \sim 3$ & \\
\hline Missing data, no. (\%) & $6(13)$ & $7(21)$ & \\
\hline
\end{tabular}


TABle 1: Continued.

\begin{tabular}{|c|c|c|c|}
\hline Characteristics & tMSLCs $(+)(N=48)$ & $\operatorname{tMSLCs}(-)(N=34)$ & $P$ value \\
\hline Ki 67 & & & 0.739 \\
\hline IHC negative (<10\%), no. $(\%)$ & $5(11)$ & $3(9)$ & \\
\hline IHC positive ( $\geq 10 \%)$, no. $(\%)$ & $40(89)$ & $31(91)$ & \\
\hline IHC $\%$ mean \pm S.D. & $22.6 \pm 14.1$ & $30.1 \pm 20.2$ & 0.057 \\
\hline Range (\%) & $2 \sim 60$ & $3 \sim 80$ & \\
\hline Missing data, no. (\%) & $3(6)$ & $0(0)$ & \\
\hline
\end{tabular}

S.D.: standard deviation; IDH: isocitrate dehydrogenase; LOH: loss of heterozygosity; OP/Stupp: operation followed by Stupp’s regimen; MGMT: O-6methylguanine-DNA methyltransferase; EGFR: epidermal growth factor receptor; NA: not available.

TABLE 2: Cox proportional hazard regression model of factors prognostic of overall survival in patients with pGBM.

\begin{tabular}{|c|c|c|c|c|c|c|}
\hline \multirow{2}{*}{ Characteristics } & \multicolumn{3}{|c|}{ Univariate } & \multicolumn{3}{|c|}{ Multivariate } \\
\hline & OR & $95 \% \mathrm{CI}$ & $P$ value & OR & $95 \% \mathrm{CI}$ & $P$ value \\
\hline Isolation of tMSLCs & 2.4 & $1.2 \sim 5.1$ & 0.017 & 2.5 & $1.1 \sim 5.6$ & 0.021 \\
\hline Age $\geq 50$ years & 1.3 & $0.5 \sim 3.1$ & 0.587 & & & \\
\hline Extent of resection & 0.7 & $0.4 \sim 1.1$ & 0.151 & 0.5 & $0.2 \sim 0.8$ & 0.011 \\
\hline IDH1 mutation & 1.6 & $0.4 \sim 6.7$ & 0.551 & & & \\
\hline LOH 1p19q & 1.3 & $0.6 \sim 3.0$ & 0.532 & 1.1 & $0.4 \sim 2.6$ & 0.869 \\
\hline MGMT methylation & 0.8 & $0.4 \sim 1.6$ & 0.522 & 0.9 & $0.4 \sim 1.9$ & 0.792 \\
\hline p $53 \geq 50 \%$ & 0.7 & $0.2 \sim 1.8$ & 0.418 & & & \\
\hline EGFR & 1.1 & $0.7 \sim 1.5$ & 0.782 & & & \\
\hline $\mathrm{Ki} 67$ index $\geq 10 \%$ & 3.3 & $0.5 \sim 24.7$ & 0.235 & 5.4 & $0.7 \sim 42.0$ & 0.107 \\
\hline
\end{tabular}

tMSLCs: tumor mesenchymal stem-like cells; IDH: isocitrate dehydrogenase; LOH: loss of heterozygosity; MGMT: O-6-methylguanine-DNA methyltransferase; EGFR: epidermal growth factor receptor; OR: odds ratio; CI: confidence interval.

statuses $P=0.057$ for the percentage of immunohistochemistry). All of 48 tMSLCs isolated from specimens showed trilineage differentiation, expression of MSC surface markers, and adherence to a plastic plate without gliomagenesis.

3.2. Patient Survival. At a medium follow-up of 11.1 months, 38 patients died. The median survival duration of all pGBM patients was 18.6 months, 15.0 months in patients positive for tMSLCs and 29.5 months in patients negative for tMSLCs $(P=0.014$; Figure 1$)$. The 6-, 12-, and 24-month actuarial rates were $86 \%, 60 \%$, and $21 \%$, respectively, in patients positive for tMSLCs and $93 \%, 79 \%$, and $61 \%$, respectively, in patients negative for tMSLCs. From univariate cox proportional regression, the only factor associated with poor survival was isolation of tMSLCs from the specimen $(\mathrm{OR}=2.4$, 95\% CI $=1.2 \sim 5.1, P=0.017$; Table 2). We included extent of resection, codeletion of 1p19q, MGMT methylation, and Ki 67 index to a multivariate cox model with a result that isolation of tMSLCs $(\mathrm{OR}=2.5,95 \% \mathrm{CI}=1.1 \sim 5.6, P=0.021)$ was associated with poorer outcome and larger extent of resection had association with better prognosis $(\mathrm{OR}=0.5,95 \% \mathrm{CI}=$ $0.2 \sim 0.8, P=0.011)$ while codeletion of $1 \mathrm{p} 19 \mathrm{q}$, MGMT methylation, and Ki 67 index does not differentiate survival of patients.

\section{Discussion}

MSLCs are cells with MSC-like properties that have been isolated from brain $[6,7]$, especially from brain tumors
(tMSLCs) [2, 9-11, 13, 38]. These cells possess MSC surface antigens, show trilineage differentiation, adhere to plastic plates, and are nontumorigenic $[11,13,35]$.

This study found that OS differed significantly between pGBM patients positive and negative for tMSLCs, suggesting that tMSLCs may play a role in the progression of pGBM. Although the "seed and soil" concept of cancer biology was proposed more than 125 years ago [39-41], GBM tumorspheres (TS) have been demonstrated recently [42, 43], with tMSLCs being a factor in this concept [9-12]. Although the exact function of tMSLCs in pGBM is not well understood, this study showed that tMSLCs were clinically important, in that they were prognostic of survival. The next step should be to assess the interactions between the GBM TS and tMSLCs.

Our overall survival (18.6 months) was higher than previously reported paper [31]. Although we described gross total resection in this paper, we indeed did supratotal resection, which made longer overall survival in our patients; however it was not proved through publication. The small number of patients ( 82 cases in total) recruited to this retrospective study is a limitation to interpret the results of this study. Although we compiled as many patients as possible, only subgroup that meets strict inclusion and exclusion criteria has limited number of patients. As our analysis does not show different prognosis in overall survival that was determined by MGMT methylation or $\mathrm{LOH}$ of $1 \mathrm{p} 19 \mathrm{q}$ which was shown in more inclusive larger patient set, cautious interpretation of our result is required. In addition, our study included IDH1 mutant patients and unknown patients, although we described 


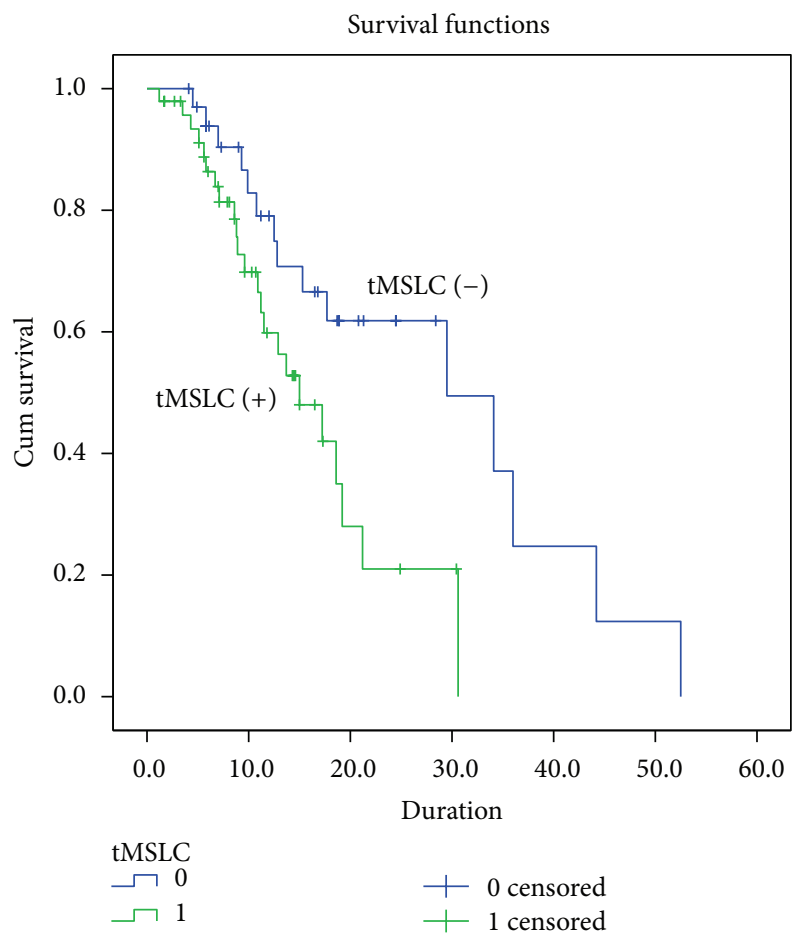

FIgURE 1: Kaplan-Meier estimates of overall survival according to tMSLCs isolation ( $P=0.014$ as calculated by the log-rank test).

pGBM. IDH1 mutant patients should be secondary GBM but these patients were allocated to both groups without statistical significance, so we included all IDH1 mutants patients and unknown patients in this study.

From original group of 82 patients, only five of 70 patients tested $(7.1 \%)$ had IDH1 mutations, including one from 27 tMSLC-negative (3.7\%) patients and four from 43 tMSLCpositive $(9.3 \%)$ patients. While these 5 tumors were not clinically suspected as secondary glioblastomas, they were excluded from multivariate cox regression model as the entity was shown to have different prognosis [37]. Patients with mutated IDH1 were younger than those with wild type IDH1 (45.3 versus 60.7 years) [37]. Although $\mathrm{LOH}$ at $1 \mathrm{p}$ or $19 q$ was found to correlate with longer OS in patients with oligodendroglioma [44], the association in patients with pGBM remains unclear. In our result, codeletion of $1 \mathrm{p} 19 \mathrm{q}$ was not associated with prognosis (univariate: $\mathrm{OR}=1.3,95 \%$ $\mathrm{CI}=0.6 \sim 3.0, P=0.532$; multivariate: $\mathrm{OR}=1.1,95 \% \mathrm{CI}=$ $0.4 \sim 2.6, P=0.869)$. Analysis of MGMT promotor showed that $42 \%$ of specimens were methylated (41\% in tMSLCs $(+)$, $47 \%$ in tMSLCs $(-))$ and that median OS tended to be longer in patients with methylation while lacking statistical significance (18.6 versus 15.0 months, $P=0.650$ ). IHC analysis of p53 found that $28 \%$ of specimens were stained for this marker. However, median OS was similar in $\mathrm{p} 53$ positive and negative patients (18.6 versus 13.7 months, $P=0.415$ ).

In this study, tMSLCs were isolated from $58.5 \%$ of patients with pGBM, compared with $46.2 \%$ in a previous study [11]. This increase, despite using the same isolation method, reemphasizes that isolation of a specific cell type from a tumor specimen requires a standardized method or may reflect a learning curve among laboratory staffs. Further research may identify specific cell markers prognostic of OS. We present novel findings of tMSLCs as a prognostic marker. Several noteworthy publications have discussed potential roles for MSLCs in glioma natural history, such as increase in angiogenesis [12] or increased proliferation and self-renewal of glioma stem cells [13]. Although we could introduce possible mechanisms based on published articles $[12,13]$, this study could not show any direct biological mechanism of tMSLCs as prognostic markers, which we believe one of the limitations of current study.

Mesenchymal features may contribute to poor survival in patients with brain tumors. Higher grade gliomas [11] and meningiomas [10] are more likely to be identified to have tMSLCs. Indeed, tMSLCs could not be isolated from WHO grade 1 gliomas and meningiomas, whereas $20 \%, 33 \%$, and $32 \%$ (or $46.2 \%$ without secondary GBM and recurrent GBM) of WHO grade 2, 3, and 4 gliomas, respectively, were positive for tMSLCs [11]. It remains unclear, however, how the presence of tMSLCs aggravates the natural history of a brain tumor or contributes to tumor progression.

\section{Conclusion}

Isolation of tMSLCs is associated with the survival of pGBM patients. tMSLCs may have a critical role in the survival of patients with pGBM. Other cell types may predict the clinical course of patients with pGBM. In addition, cell surface markers and molecular markers of pGBM may have prognostic value, and the interactions of tMSLCs with gCSCs may better reveal the role of these cell types in $\mathrm{pGBM}$ patients.

\section{Conflict of Interests}

The authors declare no conflict of interests.

\section{Authors' Contribution}

Seon-Jin Yoon and Jin-Kyoung Shim contributed equally to this work as co-first authors.

\section{Acknowledgments}

This research was supported by the Basic Science Research Program through the National Research Foundation of Korea (NRF) funded by the Ministry of Education, Science and Technology (NRF-2013R1A1A2006427) and by a grant of the Korean Health Technology R\&D Project, Ministry of Health \& Welfare, Republic of Korea (HI14C0042).

\section{References}

[1] J. Dong, Q. Zhang, Q. Huang et al., “Glioma stem cells involved in tumor tissue remodeling in a xenograft model: laboratory investigation," Journal of Neurosurgery, vol. 113, no. 2, pp. 249260, 2010.

[2] J. Behnan, P. Isakson, M. Joel et al., "Recruited brain tumorderived mesenchymal stem cells contribute to brain tumor progression," STEM CELLS, vol. 32, no. 5, pp. 1110-1123, 2014. 
[3] A. Golebiewska, S. Bougnaud, D. Stieber et al., "Side population in human glioblastoma is non-tumorigenic and characterizes brain endothelial cells," Brain, vol. 136, no. 5, pp. 1462-1475, 2013.

[4] L. Li, J. Cole, and D. A. Margolin, "Cancer stem cell and stromal microenvironment," Ochsner Journal, vol. 13, no. 1, pp. 109-118, 2013.

[5] B. Mognetti, G. La Montagna, M. G. Perrelli, P. Pagliaro, and C. Penna, "Bone marrow mesenchymal stem cells increase motility of prostate cancer cells via production of stromal cell-derived factor-1 $\alpha$, Journal of Cellular and Molecular Medicine, vol. 17, no. 2, pp. 287-292, 2013.

[6] L. da Silva Meirelles, P. C. Chagastelles, and N. B. Nardi, "Mesenchymal stem cells reside in virtually all post-natal organs and tissues," Journal of Cell Science, vol. 119, part 11, pp. 2204-2213, 2006.

[7] S.-G. Kang, N. Shinojima, A. Hossain et al., "Isolation and perivascular localization of mesenchymal stem cells from mouse brain," Neurosurgery, vol. 67, no. 3, pp. 711-720, 2010.

[8] F. F. Lang, T. Amano, N. Hata, J. Gumin, K. Aldape, and H. Colman, "Bone marrow-derived mesenchymal stem cells are recruited to and alter the growth of human gliomas," NeuroOncology, vol. 9, p. 596, 2007.

[9] J. Kwak, H.-J. Shin, S.-H. Kim et al., "Isolation of tumor spheres and mesenchymal stem-like cells from a single primitive neuroectodermal tumor specimen," Child's Nervous System, vol. 29, no. 12, pp. 2229-2239, 2013.

[10] H.-Y. Lim, K. M. Kim, B. K. Kim et al., "Isolation of mesenchymal stem-like cells in meningioma specimens," International Journal of Oncology, vol. 43, no. 4, pp. 1260-1268, 2013.

[11] Y. G. Kim, S. Jeon, G.-Y. Sin et al., "Existence of glioma stroma mesenchymal stemlike cells in Korean glioma specimens," Child's Nervous System, vol. 29, no. 4, pp. 549-563, 2013.

[12] B. H. Kong, H.-D. Shin, S.-H. Kim et al., "Increased in vivo angiogenic effect of glioma stromal mesenchymal stem-like cells on glioma cancer stem cells from patients with glioblastoma," International Journal of Oncology, vol. 42, no. 5, pp. 17541762, 2013.

[13] A. Hossain, J. Gumin, F. Gao et al., "Mesenchymal stem cells isolated from human gliomas increase proliferation and maintain stemness of glioma stem cells through the IL-6/gp130/STAT3 pathway," STEM CELLS, vol. 33, no. 8, pp. 2400-2415, 2015.

[14] E. Bourkoula, D. Mangoni, T. Ius et al., "Glioma-associated stem cells: a novel class of tumor-supporting cells able to predict prognosis of human low-grade gliomas," Stem Cells, vol. 32, no. 5, pp. 1239-1253, 2014.

[15] W. A. Freije, F. E. Castro-Vargas, Z. Fang et al., "Gene expression profiling of gliomas strongly predicts survival," Cancer Research, vol. 64, no. 18, pp. 6503-6510, 2004.

[16] Y. Liang, M. Diehn, N. Watson et al., "Gene expression profiling reveals molecularly and clinically distinct subtypes of glioblastoma multiforme," Proceedings of the National Academy of Sciences of the United States of America, vol. 102, no. 16, pp. 5814-5819, 2005.

[17] H. S. Phillips, S. Kharbanda, R. Chen et al., "Molecular subclasses of high-grade glioma predict prognosis, delineate a pattern of disease progression, and resemble stages in neurogenesis," Cancer Cell, vol. 9, no. 3, pp. 157-173, 2006.

[18] R. G. W. Verhaak, K. A. Hoadley, E. Purdom et al., "Integrated genomic analysis identifies clinically relevant subtypes of glioblastoma characterized by abnormalities in PDGFRA,
IDH1, EGFR, and NF1," Cancer Cell, vol. 17, no. 1, pp. 98-110, 2010.

[19] K. Aldape, G. Zadeh, S. Mansouri, G. Reifenberger, and A. von Deimling, "Glioblastoma: pathology, molecular mechanisms and markers," Acta Neuropathologica, vol. 129, no. 6, pp. 829848, 2015.

[20] R. Beroukhim, G. Getz, L. Nghiemphu et al., "Assessing the significance of chromosomal aberrations in cancer: methodology and application to glioma," Proceedings of the National Academy of Sciences of the United States of America, vol. 104, no. 50, pp. 20007-20012, 2007.

[21] C. Brennan, H. Momota, D. Hambardzumyan et al., "Glioblastoma subclasses can be defined by activity among signal transduction pathways and associated genomic alterations," PLoS ONE, vol. 4, no. 11, Article ID e7752, 2009.

[22] R. Shen, Q. Mo, N. Schultz et al., "Integrative subtype discovery in glioblastoma using iCluster," PLoS ONE, vol. 7, no. 4, Article ID e35236, 2012.

[23] M. Karsy, J. A. Neil, J. Guan, M. A. Mahan, H. Colman, and R. L. Jensen, "A practical review of prognostic correlations of molecular biomarkers in glioblastoma," Neurosurgical Focus, vol. 38, no. 3, p. E4, 2015.

[24] M. E. Hegi, A.-C. Diserens, T. Gorlia et al., "MGMT gene silencing and benefit from temozolomide in glioblastoma," The New England Journal of Medicine, vol. 352, no. 10, pp. 997-1003, 2005.

[25] M. Sanson, Y. Marie, S. Paris et al., "Isocitrate dehydrogenase 1 codon 132 mutation is an important prognostic biomarker in gliomas," Journal of Clinical Oncology, vol. 27, no. 25, pp. 41504154, 2009.

[26] H. Nakamura, K. Makino, and J.-I. Kuratsu, "Molecular and clinical analysis of glioblastoma with an oligodendroglial component (GBMO)," Brain Tumor Pathology, vol. 28, no. 3, pp. 185190, 2011.

[27] M. Mizoguchi, K. Yoshimoto, X. Ma et al., "Molecular characteristics of glioblastoma with $1 \mathrm{p} / 19 \mathrm{q}$ co-deletion," Brain Tumor Pathology, vol. 29, no. 3, pp. 148-153, 2012.

[28] B. H. Kong, J. H. Moon, Y.-M. Huh et al., "Prognostic value of glioma cancer stem cell isolation in survival of primary glioblastoma patients," Stem Cells International, vol. 2014, Article ID 838950, 6 pages, 2014.

[29] C. Isella, A. Terrasi, S. E. Bellomo et al., "Stromal contribution to the colorectal cancer transcriptome," Nature Genetics, vol. 47, no. 4, pp. 312-319, 2015.

[30] A. Calon, E. Lonardo, A. Berenguer-Llergo et al., "Stromal gene expression defines poor-prognosis subtypes in colorectal cancer," Nature Genetics, vol. 47, no. 4, pp. 320-329, 2015.

[31] R. Stupp, W. P. Mason, M. J. van den Bent et al., "Radiotherapy plus concomitant and adjuvant temozolomide for glioblastoma," The New England Journal of Medicine, vol. 352, no. 10, pp. 987-996, 2005.

[32] D. N. Louis, H. Ohgaki, O. D. Wiestler et al., "The 2007 WHO classification of tumours of the central nervous system," Acta Neuropathologica, vol. 114, no. 2, pp. 97-109, 2007.

[33] M. J. McGirt, K. L. Chaichana, M. Gathinji et al., "Independent association of extent of resection with survival in patients with malignant brain astrocytoma," Journal of Neurosurgery, vol. 110, no. 1, pp. 156-162, 2009.

[34] C. M. Kramm, S. Wagner, S. Van Gool et al., "Improved survival after gross total resection of malignant gliomas in pediatric patients from the HIT-GBM studies," Anticancer Research, vol. 26, no. 5, pp. 3773-3779, 2006. 
[35] M. Dominici, K. Le Blanc, I. Mueller et al., "Minimal criteria for defining multipotent mesenchymal stromal cells. The International Society for Cellular Therapy position statement," Cytotherapy, vol. 8, no. 4, pp. 315-317, 2006.

[36] A. Mathew, M. Pandey, and N. S. Murthy, "Survival analysis: caveats and pitfalls," European Journal of Surgical Oncology, vol. 25, no. 3, pp. 321-329, 1999.

[37] S. Nobusawa, T. Watanabe, P. Kleihues, and H. Ohgaki, "IDH1 mutations as molecular signature and predictive factor of secondary glioblastomas," Clinical Cancer Research, vol. 15, no. 19, pp. 6002-6007, 2009.

[38] C.-L. Tso, P. Shintaku, J. Chen et al., "Primary glioblastomas express mesenchymal stem-like properties," Molecular Cancer Research, vol. 4, no. 9, pp. 607-619, 2006.

[39] S. Paget, "The distribution of secondary growths in cancer of the breast," The Lancet, vol. 133, no. 3421, pp. 571-573, 1889.

[40] G. Poste and I. J. Fidler, "The pathogenesis of cancer metastasis," Nature, vol. 283, no. 5743, pp. 139-146, 1980.

[41] I. J. Fidler and G. Poste, "The 'seed and soil' hypothesis revisited," The Lancet Oncology, vol. 9, no. 8, p. 808, 2008.

[42] B. H. Kong, N.-R. Park, J.-K. Shim et al., "Isolation of glioma cancer stem cells in relation to histological grades in glioma specimens," Child's Nervous System, vol. 29, no. 2, pp. 217-229, 2013.

[43] E. Sulman, K. Aldape, and H. Colman, "Brain tumor stem cells," Current Problems in Cancer, vol. 32, no. 3, pp. 124-142, 2008.

[44] K. B. Fallon, C. A. Palmer, K. A. Roth et al., "Prognostic value of 1p, 19q, 9p, 10q, and EGFR-FISH analyses in recurrent oligodendrogliomas," Journal of Neuropathology and Experimental Neurology, vol. 63, no. 4, pp. 314-322, 2004. 

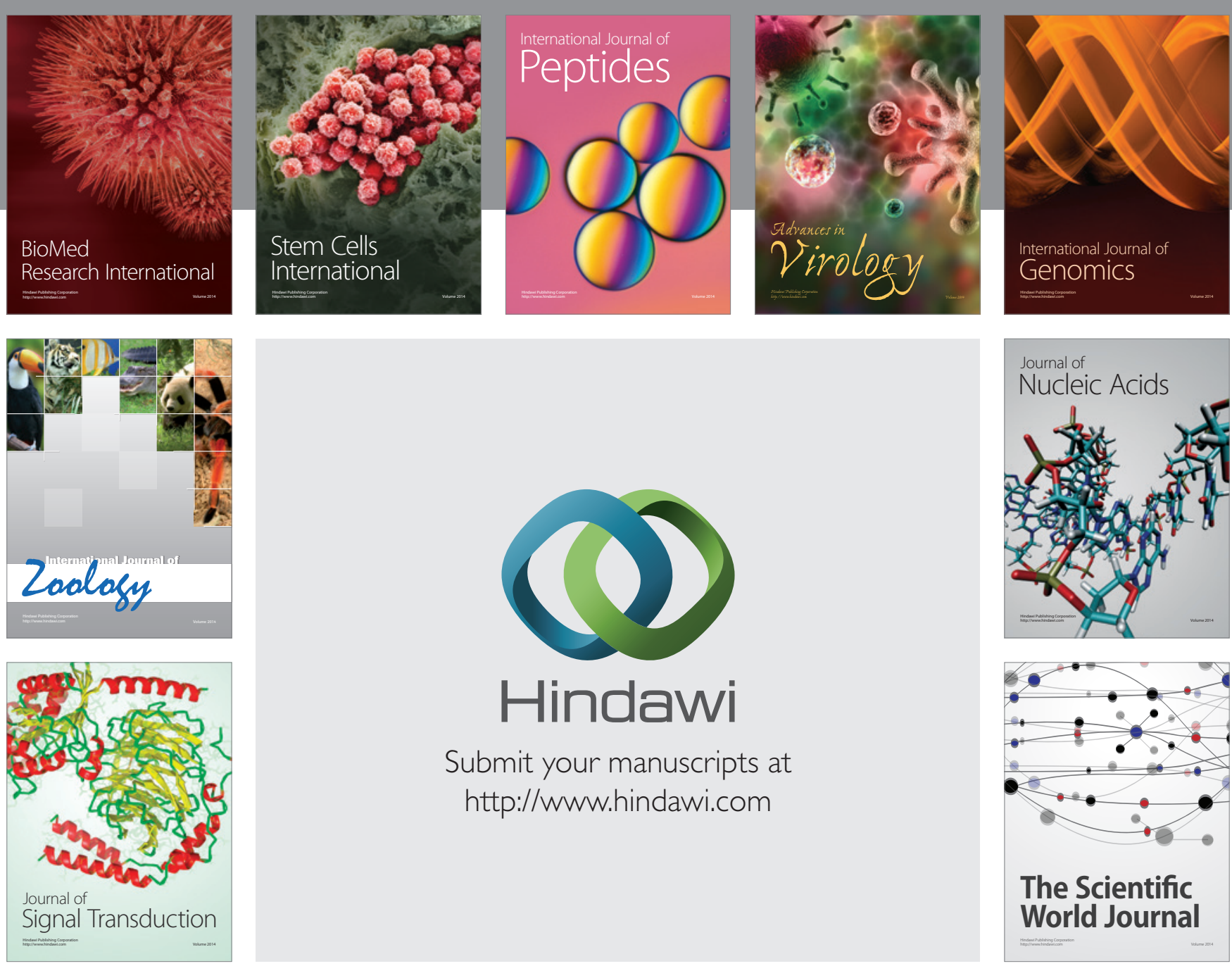

Submit your manuscripts at

http://www.hindawi.com
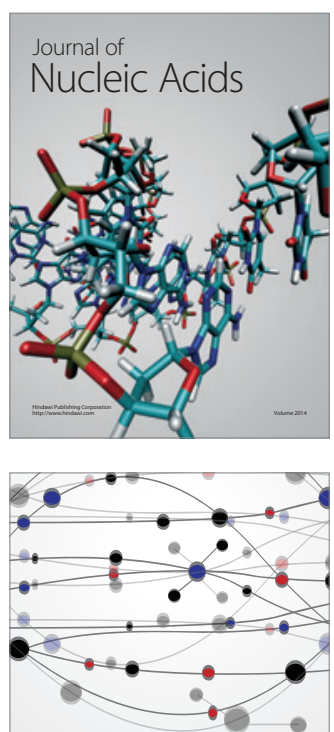

The Scientific World Journal
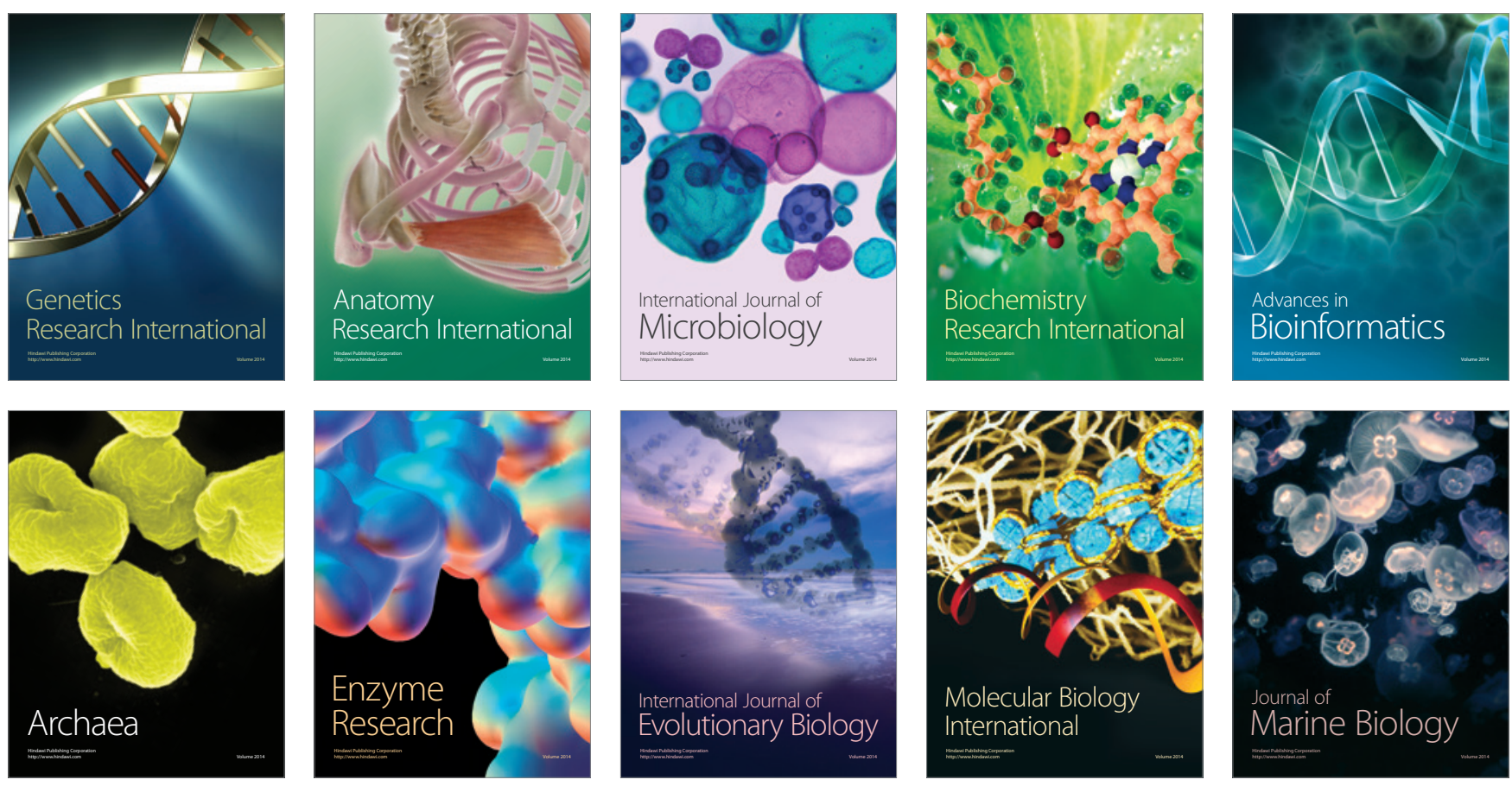